\title{
William Wordsworth y sus traductores franceses
}

SOLEDAD Díaz ALARCón
Universidad de Córdoba

Fecha de recepción: 5 de febrero de 2009

Fecha de aceptación: 15 de abril de 2009

Resumen: Trabajo que analiza la influencia de los poetas 'lakistas' en el escritor francés Saint-Beuve, fundamentalmente de Wordsworth, y en el que se estudian tres de los sonetos imitados: "I am not one who much or oft delight", "Scorn not the sonnet" y "It is a beauteous evening, calm and free". En un segundo término la de otros traductores franceses amantes y difusores en Francia de la poesía inglesa de la segunda mitad del XVIII y principios del XIX: Émile Legouis y André Koszul.

Palabras clave: Poesía inglesa, Imitación, Sainte-Beuve

Abstract: In this paper I discuss firstly the influence of the 'lakist' poets, and mostly Wordsworth, on the French writer Saint- Beuve. Then three imitated sonnets are surveyed: "I am not one who much of oft delight", "Scorn not the sonnet" and "It is a beauteous evening, calm and free". Secondly, I turn my attention to other French translators and other lovers of English poetry, such as Émile Legouis and André Koszul, who contributed to its spreading in the second half of the 18th century and beginnings of the next century.

Key words: English poetry, Imitation, Sainte-Beuve

INTRODUCCIÓN

Es un tópico innegable en la literatura mundial la influencia que los poetas 'lakistas' han tenido en el desarrollo de la lírica occidental contemporánea, y en el movimiento romántico en particular; de hecho, este grupo de poetas ingleses componen entre 1798 y 1815 los primeros poemas de tendencia claramente romántica ${ }^{1}$ alejándose de las preceptivas barrocas y neoclásicas y que influyeron no sólo a aquellos autores que vivieron en "The Lake District", sino a una ingente cantidad de poetas que escribirían sus obras en el siglo XIX. El término "The Lake Poets" surge por primera vez en agosto de 1817, de la mano del crítico y fundador de la Edinburgh Review ${ }^{2}$ Francis Jeffrey, para referirse a los escritores Robert

\footnotetext{
${ }^{1}$ Aunque buena parte de la crítica sitúe ya en esa órbita los poemas de los denominados poetas prerrománticos y los de la "Graveyard School" o poesía de los cementerios.

2 The Edinburgh Review fue una de las revistas británicas más influyentes del siglo XIX. Adoptó como lema "absolvitur de nocens d'ubi de damnatur de judex» ("El juez es condenado cuando el culpable es absuelto") de Publilius Syrus. Fue creada el 10 de octubre de 1802 por
} 
Southey, Samuel Taylor Coleridge y William Wordsworth, que vivieron en la región de los Lagos al noroeste de Inglaterra y enriquecieron y renovaron la poesía a través de una vuelta a la naturaleza, al retrato de los humildes y a la práctica de la balada popular. En concreto, el prólogo que Wordsworth escribió en 1800 para la segunda edición de su obra Lyrical Ballads, publicada por primera vez en 1798, se convirtió en un verdadero manifiesto de la lírica romántica, ya que dejaba constancia de los aires renovadores que afectaban a la poesía, definiéndola como "the spontaneous overflow of powerful feelings"3, donde Wordsworth defiende el lenguaje coloquial y los hechos de la vida cotidiana ${ }^{4}$.

Resumamos este breve acercamiento al poeta inglés, poniendo de relieve que su poesía ya no levanta discusiones ni controversias, habiéndose convertido en uno de esos casos raros en la literatura mundial en que llega a ser ejemplo y paradigma de un tipo de poesía prácticamente insuperable, igual que ocurre con otros poetas cuyo reconocimiento trasciende a su propia obra (Baudelaire, Rimbaud, Dickinson, Whitman, etc.).

\section{SAINTE-BEUVE, EL “DISCÍPULO” FRANCÉS (1804-1869)}

Los cambios y las nuevas ideas que Wordsworth introduce en una lírica, seducida por las normas que marca la prosa, se dejan sentir rápidamente en Francia, y más concretamente en la creación de Charles Augustin Sainte-Beuve, que con sus obras Les Consolations y Pensées d'août, quiere reivindicar lo que él entiende como un "lakisme français", y se convierte en uno de los primeros traductores europeos de William

Francis Jeffrey, Sydney Smith y Henry Brougham y editada trimestralmente por Archibald Constable hasta 1929.

3 "El espontáneo desbordamiento de intensas emociones", definición que podemos encontrar en el libro de William Wordsworth, Preface to Lyrical Ballads, 1800, 1802: Edición Bilingüe, Madrid: Ediciones Hiperion, S.L., 1999.

${ }^{4}$ En el famoso Prólogo llevará a cabo, como en todo el resto de su obra, un vigoroso y lúcido esfuerzo por llevar a la poesía las vibraciones más sutiles del alma, sirviéndose de los elementos que proporciona la vida cotidiana, transformados por la intervención de una sensibilidad extremadamente imaginativa. Lo familiar y sencillo, lo trivial y habitual, alcanzan en los poemas de Wordsworth un resplandor especial, como si al atravesar el alma del poeta se hubieran impregnado de un misterioso aroma. En la publicación conjunta de Lyrical Ballads (1798), Wordsworth y Coleridge pretendían cambiar el signo bajo el cual había crecido la poesía inglesa. Tuvieron precursores, ciertamente, pero ninguno se atrevió a proclamar, como lo hizo Wordsworth en el prólogo de esta obra, que "el principal objeto propuesto con estos poemas era escoger incidentes y situaciones de la vida ordinaria y relatarlos o describirlos completamente y, en tanto como fuera posible, en una selección de lenguaje realmente usado por los hombres, y al mismo tiempo verter sobre aquéllos un cierto colorido de la imaginación, por el cual las cosas corrientes serían presentadas al espíritu con inusitado aspecto". 
Wordsworth y de Colerige ${ }^{5}$. La complejidad de la obra de Sainte-Beuve pone de manifiesto la impresión 'meándrica' de su propia vida, que dedicará por entero a la literatura, desarrollando así una producción que pasará por la poesía, la crítica y la historia, sin olvidar su colaboración con la prensa o su considerable correspondencia que no hace más que testimoniar las relaciones del escritor con sus contemporáneos y su público. La obra de Sainte-Beuve fue menospreciada casi desde su gestación, siendo tan sólo reconocida su faceta como crítico ${ }^{6}$ impregnada de romanticismo y deudora del clasicismo formalista, contrario a todo exceso y desequilibrio aparente, y marcado por el cientificismo y el realismo. Sainte-Beuve no sólo será el crítico y biógrafo más importante de su época, sino un poeta aceptable y un pulcro e inteligente novelista como demuestra en sus obras Vie, Poésies et Pensées de Joseph Delorme (1829), primera colección poética donde combinará la novela biográfica, el lirismo y las reflexiones críticas. En ella, los poemas forman un caleidoscopio de colores y de temas diferenciados: languidez elegíaca, armonía lastimera de inspiración lamartiniana, sonetos (estrofa que Sainte-Beuve recupera del olvido al que había estado relegada durante el siglo XVIII), o sonoras estrofas al estilo de Ronsard y de Victor Hugo. Pero será sin duda en Les Consolations (1830), donde el poeta dé rienda suelta a la confidencia, y muestre su lado más íntimo, siendo Pensées d'août (1837), en las que el tono narrativo va ganando terreno, donde anuncie las alteraciones a las que somete el poema, que se ve arrastrado y sometido a los dictámenes de la prosa. Con Sainte-Beuve nos encontramos ante un poeta 'sin retórica', muy próximo al lenguaje cotidiano y de clara influencia 'lakista" y 'wordsworthiana', poesía en la que SainteBeuve se basa para reivindicar un lirismo simple, sincero y realista, exaltando la naturaleza y el hogar, las alegrías y las penas cotidianas. Admirado por Paul Verlaine, Sully Prudhomme y François Coppée, podemos constatar igualmente que Sainte-Beuve es uno de los padres del denominado 'neo-intimismo' que surgiría hacia 1865. Creemos, con razones fundadas en el estudio de su obra, que su realismo poético -en el que el sentido del arte y de la moral atemperan la representación de lo real- habría ejercido, sin duda, una mayor influencia si no se hubiese comprometido con una religiosidad heredada del romanticismo católico.

Debuta como crítico con un estudio histórico, Tableau historique et critique de la poésie française et du théâtre français au XVI siècle (1829),

\footnotetext{
${ }^{5}$ Antes de traducir las obras de los 'lakistas' Sainte-Beuve ya lo había hecho con la poesía del poeta 'intimista' William Cowper.

${ }^{6}$ Que hoy tampoco se considera muy afortunada al basarse únicamente en la biografía de los autores estudiados; casi puede afirmarse, sin caer en el error, que Sainte-Beuve 'tuvo la desgracia' de vivir en una época en la que proliferaron grandes genios poéticos (Lamartine, Hugo, Vigny...).
} 
aparecido previamente en la revista Le Globe, obra que se sitúa entre los estudios más notables de su tiempo y que le abrirá las puertas de revistas en las que no dejaría de colaborar, como La Revue de Paris, y con la que inaugura el género de los 'retratos literarios'. Ahí comienzan a aparecer sus lecturas de las vidas y obras de Boileau, La Fontaine, André Chénier, Racine y numerosos otros, recogidas entre 1832 y 1839 en cinco volúmenes. Durante su estancia en Suiza (1837-1838), donde es profesor en la Academia de Lausana, inicia una de sus obras más notables, PortRoyal, cuyo sexto y último volumen es publicado en 1867. Elegido miembro de la Academia en 1844, cinco años más tarde -mientras ejerce como 'Professeur' en el Collège de France-, inicia en el diario Le Constitutionnel la serie de artículos que constituirán los quince volúmenes de sus famosas Causeries du Lundi ${ }^{7}$.

Tras esta sucinta introducción a la obra de Sainte-Beuve, en la que hemos querido dejar constancia de la pretensión del autor francés de aclimatar en el país galo la poesía íntima y familiar de uno de los tres poetas 'lakistas', dediquemos nuestra atención a las traducciones e imitaciones de la poesía de William Wordsworth, que Sainte-Beuve realiza y publica en varias de sus obras, reunidas en octubre de 1863 en un sólo volumen con el título de Poésies Complètes ${ }^{8}$, y que dividirá en dos partes, porque, como indica en el "Avertissement" de dicha obra, constituían "deux veines très distinctes": "la veine de Joseph Delorme", ardiente, positiva, real, a veces sensual o al menos natural, tanto en la inspiración como en la expresión; y

\footnotetext{
${ }^{7}$ Entre sus obras más notables mencionemos: Chateaubriand et son groupe littéraire sous l'Empire (1861), Nouveaux Lundis (1868) o Portraits des femmes (1844 y 1870).

Aclaremos que en su faceta de crítico, fundamentalmente en su juventud, Sainte-Beuve piensa que para comprender una obra hay que comprender al autor, a la persona, pero la persona era más fácil de entender gracias, precisamente, a su obra y pide para cierta poesía y narrativa de XVIII responsabilidad moral y en las novelas de los románticos, una forma equilibrada. En plena eclosión del romanticismo francés, buscó un contrapeso en el clasicismo. Pesimista, con el paso de los años se alejaría de la creencia de la misión del poeta en el sentido romántico, acercándose, en cambio, a la juiciosa y constructiva misión del crítico moralista, tal como lo fueron algunos ideólogos del siglo XVII: pasión por iluminar, instruir, y ensanchar el corazón y la razón. Defendió la obra de arte desde una perspectiva que evoca por momentos los preceptos románticos, aunque siempre desde un gusto refinado y una tendencia al equilibrio que le impedía, a pesar de ver su talento, aceptar del todo como grandes las obras de Balzac o Hugo. Como quiera que sea, es innegable que la riqueza y la unicidad de la obra 'beuviana' nacen de las contradicciones que predominan en ella y de la pasión que la anima: el deseo de saber. Para llegar a este conocimiento despliega una compleja pluralidad de enfoques: crítica estilística, identificación intuitiva, o historicismo matizado. Formado como neoclásico, de inspiración romántica, epicúreo impresionista, anunciador del positivismo, Sainte-Beuve refleja las corrientes que comparten la crítica literaria del siglo XIX; poeta, biógrafo, historiador, ensayista, moralista, manteniéndose en el cruce de caminos de todas las críticas actuales.

${ }^{8}$ Poésies complètes de Sainte-Beuve, Paris: Charpentier Libraire-Éditeur, 1845, edición que hemos seguido para nuestro trabajo.
} 
"la veine Consolations", más mística, más ideal, religiosa y sublime. Tanto en una como en otra encontramos una serie de poemas con la anotación "imité de Wordsworth", que citamos a continuación:

En Vie, poésies et pensées de Joseph Delorme se recogen los siguientes:

1. «Le plus long jour de l'année. À Laure» (p.80)

2. «Je ne suis pas de ceux pour qui les causeries» (p. 113)

3. «Ne ris point des sonnets, ô critique moqueur!» (p.113)

En Consolations encontramos tres sonetos:

1. Sonnet XIII «C'est un beau soir, un soir paisible et solennel» (p. 206)

2. Sonnet XIV «Les passions, la guerre; une âme en frénésie» (p. 207)

3. Sonnet XV «Quand le Poète en pleurs, à la main une lyre» (p. 208)

Y en Pensées d'août, hallamos otros tres:

1. Sonnet I «Reposez-vous et remerciez. (Au sommet du Glenroe)» (p. 304)

2. Sonnet II «La Cabane du Highlander» (p. 305)

3. Sonnet III «Le Château de Bothwell» (p. 306)

Dado que para nuestro trabajo sería muy extenso el análisis de los nueve sonetos de Sainte-Beuve, 'imités' de Wordsworth, creemos que los tres elegidos son suficientemente representativos y que se encuentran además entre los más conocidos del poeta inglés: "Je ne suis pas de ceux pour qui les causeries", "Ne ris point des sonnets, ô critique moqueur!" y "C'est un beau soir, un soir paisible et solennel".

Veamos el soneto de Wordsworth, I AM NOT ONE WHO MUCH OR OFT DELIGHT y la imitación del poeta francés:

I am not One who much or oft delight to season my fireside with personal talk, about Friends, who live within an easy walk, or Neighbours, daily, weekly, in my sight:

And, for my chance-acquaintance, Ladies bright, Sons, Mothers, Maidens withering on the stalk, these all wear out of me, like Forms, with chalk painted on rich men's floors, for one feast-night.

Better than such discourse doth silence long, long, barren silence, square with my desire; to sit without emotion, hope, or aim,

By my half-kitchen my half-parlour fire, and listen to the flapping of the flame, or kettle, whispering it's faint undersong. 
Je ne suis pas de ceux pour qui les causeries, au coin du feu, l'hiver, ont de grandes douceurs; car j'ai pour tous voisins d'intrépides chasseurs, rêvant de chiens dressés, de meutes aguerries,

Et des fermiers causant jachères et prairies, et le juge de paix avec ses vieilles sœurs, deux revêches beautés parlant de ravisseurs, portraits comme on en voit sur les tapisseries.

Oh ! Combien je préfère à ce caquet si vain, tout le soir, du silence, - un silence sans fin; être assis sans penser, sans désir, sans mémoire;

Et, seul, sur mes chenets, m'éclairant aux tisons, écouter le vent battre, et gémir les cloisons, et le fagot flamber, et chanter ma bouilloire!

En una primera lectura, podemos constatar en este soneto que el poeta francés mantiene la estructura básica de este tipo de estrofa, compuesta por catorce versos "alexandrins", y en cuyos cuartetos reproduce la rima "riche embrassée" (ABBA ABBA) al igual que el soneto de Wordsworth; sin embargo, apreciamos que Sainte-Beuve varía la rima de los tercetos, ajustándose más a la composición francesa, con rima "plate" en los versos 9, 10 (CC) y "embrassée" en el resto de los tercetos (D EED); diferenciándose así de la rima de los tercetos propuesta por Wordsworth (CDE DEC). Otro aspecto que apreciamos es que no se trata de una traducción literal, como indica el autor francés con el calificativo de "imité", ya que descubrimos las diferencias más evidentes en los cuartetos:

- en primer lugar Wordsworth menciona un número mayor de grupos de personas introducidas con mayúscula (Friends, Neighbours, Ladies, Sons, Mothers, Maidens), que Sainte-Beuve simplifica haciendo sólo alusión a "voisins, fermiers, juge de paix, sœurs" en minúscula.

- del mismo modo, encontramos importantes cambios léxicos en los versos 3, 4, 5, 6: Sainte-Beuve introduce diferentes personajes (chasseurs, fermiers, juge de paix, sœurs) y de los que nos cuenta sus únicos intereses y los temas habituales en sus conversaciones. Sin embargo, Wordsworth es más genérico, centrándose más en el hecho de la cercanía y proximidad de este grupo de personas, confiriéndole así el calificativo de "Friends or Neighbours".

No obstante, las diferencias se diluyen a medida que avanzamos en la lectura del poema de Sainte-Beuve, ya que las coincidencias entre los 
tercetos de éste último y los del poema de Wordsworth son muy evidentes y nos hacen pensar más en una traducción que en una imitación.

En lo que a contenido se refiere, Sainte-Beuve nos habla en dicho poema sobre sus gustos, ofreciendo una estructura bastante clara: en los dos cuartetos, el poeta francés nos describe situaciones y compañías que rechaza y presenta con desprecio a aquellas personas, vecinos o amigos, que en dicho ambiente campestre le ofrecen conversación, y que según la opinión del poeta es absolutamente banal y falta de interés, hecho que nos lo muestra con el uso de términos "causeries, causant, caquet". Cuando introduce a sus vecinos, mejor dicho sus únicos vecinos ("j'ai pour tous voisins"), insiste en la idea de totalidad con el adjetivo "tous", por lo que no le queda esperanza de que "alguien" pueda salvarse de esta negativa consideración: "d'intrépides chasseurs", "des fermiers", "et le juge de paix". Por otra parte y gracias a la descripción que realiza de los dos primeros grupos, el poeta nos da evidentes signos de que el contexto es el campo, descrito en los versos $3,4,5$. El último grupo de personas a las que hace referencia "les vieilles sœurs du juge de paix", las ridiculiza reforzando la idea del paso del tiempo, de la vejez: "vieilles, revêches beautés, tapisseries", además de sugerirnos con los términos "parlant de ravisseurs, portraits comme on en voit sur les tapisseries" la imagen clásica del rapto de las Sabinas, jóvenes y hermosas mujeres de la tribu de los sabinos raptadas por deseosos romanos, subrayando así, la fantasía y falta de racionalidad de la conversación de dichas señoras. Todas estas opiniones las introduce desde el primer verso a través de la negación en primera persona "je ne suis pas de ceux", y explica sus razones partiendo de la partícula "car". De este modo, el poeta rechaza una idea bastante generalizada de lo agradable que es, en invierno, en el calor de la chimenea, compartir ideas o sentimientos con otras personas en placentera charla.

Al tratarse de un poema muy descriptivo, la mayor parte de los sustantivos van enriquecidos con adjetivos calificativos: "grandes douceurs, intrépides chasseurs, chiens dressés, meutes aguerries...". A las ideas expuestas en los cuartetos, contrapone lo que el poeta ama y prefiere, explicado con énfasis en los tercetos. En ellos nos muestra nítidamente tres aspectos:

- Expresión de un fuerte sentimiento por medio de la admiración "Oh!" y de los pronombres personales y adjetivos posesivos en primera persona: "Je préfère", "mes chenets", "m'éclairant", "ma bouilloire".

- El deseo de silencio y soledad, reforzado por las aliteraciones del sonido Is/ en los versos 10 y 11: "soir", "silence", "silence", "sans", "assis", "sans penser", "sans désir, "sans..." y de los sonidos nasales en el segundo terceto: "éclairant, tisons, vent, cloisons, flamber, chanter". En el primer terceto, el silencio es calificado de absoluto e infinito, subrayado por la idea 
de que dicho silencio debe abarcar y afectar no sólo al lenguaje, sino a la mente ("sans penser, sans mémoire") y al corazón ("sans désir"), conectando así con el sentimiento y el deseo del olvido. Los únicos sonidos que el poeta desea oír, en soledad ("seul" separado por comas) son los sonidos naturales y propios del hogar ("le vent battre, gémir les cloisons, le fagot flamber, chanter ma boulloire").

- El gusto por lo familiar y cotidiano expresado mediante utensilios caseros y sonidos habituales y propios del hogar. Con las personificaciones de los dos últimos versos, "gémir les cloisons", "chanter ma bouilloire" se hace más evidente que el poeta prefiere la compañía y "la conversación" de objetos familiares a la de personas: "gémir" expresa un sentimiento de tristeza y "chanter" de alegría. Concluiremos este apartado destacando la anáfora del último terceto, donde todos los versos van a ser introducidos por el sonido "e" .

\section{Segundo soneto: SCORN NOT THE SONNET}

Scorn not the Sonnet; Critic, you have frowned, mindless of its just honours; with this key Shakespeare unlocked his heart; the melody of this small lute gave ease to Petrarch's wound;

A thousand times this pipe did Tasso sound;

\footnotetext{
${ }^{9}$ Con el fin de que se puedan captar todos los matices expresados, así como para llevar a cabo un ejercicio más completo de literatura comparada veamos a continuación la traducción española del soneto del autor francés, en la que hemos intentado respetar el metro, pero sin tener en cuenta la rima, que nos obligaría a una rigidez que generalmente no conviene a la traducción poética:

"Je ne suis pas de ceux pour qui les causeries, No soy de los que creen que las conversaciones au coin du feu, l'hiver, ont de grandes douceurs; junto al fuego, en invierno, sean muy placenteras; car j'ai pour tous voisins d'intrépides chasseurs, pues tengo de vecinos tan sólo a cazadores, rêvant de chiens dressés, de meutes aguerries, que piensan sólo, intrépidos, en perros y jaurías,

Et des fermiers causant jachères et prairies, et le juge de paix avec ses vieilles sœurs, deux revêches beautés parlant de ravisseurs, portraits comme on en voit sur les tapisseries.

Oh! combien je préfère à ce caquet si vain, tout le soir, du silence, - un silence sans fin; être assis sans penser, sans désir, sans mémoire;

Et, seul, sur mes chenets, m'éclairant aux tisons, écouter le vent battre, et gémir les cloisons, et le fagot flamber, et chanter ma bouilloire!"

y granjeros que charlan del campo y sus labores, o el juez de paz y sus solteronas hermanas, dos hirsutas bellezas que sueñan con raptores, son los retratos típicos de las tapicerías.

¡Cuán mejor, yo prefiero a este cacareo vano, una tarde en silencio, un silencio infinito; sentado, sin pensar, sin deseos, sin recuerdos!; oír golpear el viento y crujir las paredes, el arder de gavillas y pitar mi tetera!
} 
with it Camöens soothed an exile's grief; the Sonnet glittered a gay myrtle leaf amid the cypress with which Dante crowned

His visionary brow: a glow-worm lamp, it cheered mild Spenser, called from Faery-land to struggle through dark ways; and, when a damp

Fell round the path of Milton, in his hand the Thing became a trumpet; whence he blew soul-animating strains--alas, too few!

Ne ris point des sonnets, ô Critique moqueur! par amour autrefois en fit le grand Shakespeare; c'est sur ce luth heureux que Pétrarque soupire, et que le Tasse aux fers soulage un peu son cœur;

Camoens de son exil abrège la longueur, car il chante en sonnets l'amour et son empire; Dante aime cette fleur de myrte, et la respire, et la mêle au cyprès qui ceint son front vainqueur;

Spenser, s'en revenant de l'île des féeries, exhale en longs sonnets ses tristesses chéries; Milton, chantant les siens, ranimait son regard:

Moi, je veux rajeunir le doux sonnet en France; Du Bellay, le premier, l'apporta de Florence, et l'on en sait plus d'un de notre vieux Ronsard.

Al igual que en el soneto anterior, Sainte-Beuve, realiza esta composición en versos "alexandrins" (12 sílabas) estructurados en dos cuartetos de rima "riche embrassée" (ABBA ABBA) y dos tercetos con rima "plate" en los versos 9 y 10 (CC), concluyendo el poema con cuatro versos de rima "embrassée" (DEED). En primer lugar, lo que llama la atención en este soneto es el comienzo tan categórico y enérgico. Sainte-Beuve se dirige en el primer verso a su interlocutor, el crítico literario ("Critique"), que mediante el uso de la mayúscula hace referencia a toda la crítica literaria. A éste, en el mismo verso, lo insulta ("moqueur") y le exige respeto por el soneto, utilizando una frase imperativa negativa y una negación absoluta ("Ne ris point des sonnets"). El resto del poema es la demostración de la dignidad y categoría de dicha estrofa utilizando como ejemplos el empleo que de ella hicieron los más grandes poetas de la literatura universal, maestros y referentes absolutos que marcaron las pautas de la creación 
poética en sus respectivas épocas: Dante, Petrarca, Shakespeare, El Tasso, Camoens, Spenser, Du Bellay, Ronsard y Milton. Señalemos que, tanto Du Bellay como Ronsard sólo aparecen en el poema de Sainte-Beuve y no en el de Wordsworth; este último dedica cada uno de los tercetos a un autor inglés: el primer terceto a Spenser y el segundo a Milton. Sin embargo Sainte-Beuve, reúne a ambos autores en el primer terceto y dedica, de este modo, el último terceto a la contribución de los autores franceses, reivindicando el uso del soneto y demostrando que las dos figuras del Renacimiento francés, Du Bellay y Ronsard, creadores del movimiento literario de La Pléiade, ya exaltaron esta estrofa y la incorporaron a sus creaciones, destacando su valor y calidad. Sainte-Beuve nombra a múltiples y notables poetas del pasado, introduciendo al primero, Shakespeare, en el primer verso con el adverbio de tiempo "autrefois". Sin embargo, nos habla de las creaciones de todos ellos no sólo en pasado sino también en presente con la pretensión de realzar su actualidad y vigencia, de ahí que de los verbos del poema, sólo tres estén en pasado "fit, ranimait, apporta"; el resto está en presente "soupire, soulage, abrége, chante, aime, respire, mêle, ceint, exhale, veux". Resaltemos, asimismo, que la palabra "sonnet" aparece en cada una de las estrofas: versos 1, 6, 10 y 12. Por otra parte, cabe constatar como a través de metáforas, Sainte-Beuve hace alusión a las obras más relevantes de cada uno de estos poetas que en su día decidieron optar por una estrofa como el soneto para cantar sus anhelos, sus amores, sus tristezas y cuya genialidad les permitirá alcanzar la inmortalidad:

- Shakespeare: su obra The Sonnets, constituye una colección de poemas cuya forma estrófica es el soneto inglés y que abordan un amplio abanico de temas tales como el amor, la belleza, la política y la mortalidad. Fueron escritos a lo largo de varios años y finalmente publicados en 1599. Además de situarse al final de la tradición sonetística petrarquista, los sonetos de este autor son considerados el comienzo de un nuevo tipo de moderna poesía amorosa.

- Petrarca: poeta y humanista italiano, considerado el primero y uno de los más importantes poetas líricos modernos. Su perfeccionamiento del soneto influyó en numerosos poetas posteriores, desde los españoles Garcilaso de la Vega y Quevedo hasta los ingleses William Shakespeare y Edmund Spenser. La más célebre de sus obras es una colección de sonetos y odas, Rime in vita e morta di Madonna Laura (posterior a 1327), ampliada años más tarde y que se conoce actualmente como Canzoniere, donde el poeta canta su amor no correspondido por Laura en un italiano vernáculo extremadamente melodioso y refinado.

- Tasso, que al igual que Petrarca, libera sus pasiones componiendo sonetos de amor, en su obra Rime. Poeta italiano de la época de la 
Contrarreforma y autor de un extenso poema épico La Gerusalemme Liberata y posteriormente De la Gerusalemme Conquistata.

- Camoens, considerado uno de los mayores poetas en lengua portuguesa, situándose su obra entre el clasicismo y el manierismo. El uso de paradojas en algunos de sus sonetos, como el conocido Amor é fogo, anuncia ya el barroco emergente.

- Dante, compositor de La Divina Commedia, obra que constituyó la base del pensamiento moderno. Actualmente es considerada la mayor obra literaria compuesta en italiano y obra maestra de la literatura universal. En el poema, figuras retóricas como la metáfora "cette fleur de myrte" (el mirto, antiguo símbolo del amor y de la belleza), la metonimia y las personificaciones "au cyprès qui ceint son front vainqueur" sirven a SainteBeuve para recordar la imagen de Dante como poeta laureado.

- Spenser, el autor de The Faerie Queene puso de moda un tipo de verso, actualmente denominado "estrofa spenseriana". En el verso que SainteBeuve dedica a este autor, donde dice textualmente "s'en revenant de l'île des féeries", hace clara alusión a dicha obra. Spenser marcó una destacada influencia en dos poetas de gran relevancia de la literatura posterior, en concreto John Milton y John Keats.

- Milton, poeta inglés conocido especialmente por su poema épico Paradise Lost, es una de las figuras más importantes del panorama literario inglés, siendo en ocasiones situado al mismo nivel que Shakespeare. La influencia de Milton en la literatura posterior se debe sobre todo a la difusión del verso blanco en poesía, (o pentámetro yámbico, de métrica regular y sin rima), especialmente en el Romanticismo.

- Du Bellay y Ronsard en 1549, forman junto a otros amigos un grupo literario llamado La Brigade, que en 1553 pasará a llamarse La Pléiade. Dicho grupo desea definir la nuevas reglas poéticas, que quedarán recogidas en la obra teórica de Du Bellay, La Défense et Illustration de la langue française. Du Bellay publicará una colección de sonetos, L'Olive, "à la manière" de Petrarca y en 1558, a su vuelta de una estancia en Roma que se prolongaría durante cuatro años, publica Les Regrets, colección de 191 sonetos en "alexandrins". Ronsard, jefe de la nueva escuela poética de La Pléiade, escribe sus Amours en 1552 colección de sonetos dedicados a Cassandre y a Marie, donde contribuye a fijar las normas del soneto regular e impone la alternancia de rimas masculinas y femeninas. En Amours de Cassandre, Amours de Marie y Sonnets pour Hélène (1578), al igual que Du Bellay, recupera la inspiración petrarquista. Cuando en el verso 13 del poema de Sainte-Beuve, se alude a la ciudad de "Florence", el autor alude a la ciudad que vio nacer a Dante, cuna del Renacimiento italiano.

Estos grandes maestros inspiran al poeta Sainte-Beuve a crear sus propios sonetos, de ahí que comience el segundo terceto con el pronombre 
en primera persona: "Moi", con la pretensión y objetivo de recuperar y como él mismo indica "rajeunir" (rejuvenecer) esta estrofa para la poesía francesa, demostrándole así al "Critique moqueur" que las razones que le llevan a burlarse del soneto son fácilmente rebatibles ${ }^{10}$.

Tercer soneto: IT IS A BEAUTEOUS EVENING, CALM AND FREE

IT is a beauteous evening, calm and free, the holy time is quiet as a Nun breathless with adoration; the broad sun is sinking down in its tranquillity;

The gentleness of heaven broods o'er the Sea: listen! the mighty Being is awake, and doth with his eternal motion make a sound like thunder--everlastingly.

Dear Child! dear Girl! that walkest with me here, if thou appear untouched by solemn thought, thy nature is not therefore less divine:

Thou liest in Abraham's bosom all the year; and worship'st at the Temple's inner shrine, God being with thee when we know it not.

C'est un beau soir, un soir paisible et solennel; à la fin du saint jour, la Nature en prière se tait, comme Marie à genoux sur la pierre, qui tremblante et muette écoutait Gabriel:

Ne ris point des sonnets, ô Critique moqueur! ¡No te rías del soneto, oh Crítico burlón! par amour autrefois en fit le grand Shakespeare; antaño por amor, los compuso el gran Shakespeare; c'est sur ce luth heureux que Pétrarque soupire, con este laúd dichoso, Petrarca suspiró, et que le Tasse aux fers soulage un peu son cœur; y el Tasso con sus hierros, su alma un poco alivió;

Camoens de son exil abrège la longueur,

Camoens de su destierro el período acortó, car il chante en sonnets l'amour et son empire; pues cantó en sonetos el imperio y su amor; Dante aime cette fleur de myrte, et la respire, Dante ama esta flor de mirto, al respirarla, et la mêle au cyprès qui ceint son front vainqueur; mezclada al laurel que ciñe su invicta frente;

Spenser, s'en revenant de l'île des féeries, Spenser, mientras volvía de la isla de las hadas exhale en longs sonnets ses tristesses chéries; sus queridas tristezas vierte en largos sonetos; Milton, chantant les siens, ranimait son regard: al recitar los suyos muestra su alegría Milton:

Moi, je veux rajeunir le doux sonnet en France; yo resucitar quiero el soneto en Francia Du Bellay, le premier, l'apporta de Florence, Du Bellay, el primero, lo trajo de Florencia et l'on en sait plus d'un de notre vieux Ronsard y tenemos más de uno de nuestro gran Ronsard. 
La mer dort; le soleil descend en paix du ciel; mais dans ce grand silence, au-dessus et derrière, on entend l'hymne heureux du triple sanctuaire, et l'orgue immense où gronde un tonnerre éternel.

Ô blonde jeune fille, à la tête baissée, qui marches près de moi, si ta sainte pensée semble moins que la mienne adorer ce moment,

C'est qu'au sein d'Abraham vivant toute l'année, ton âme est de prière, à chaque heure, baignée; c'est que ton cœur recèle un divin firmament.

Iniciemos el comentario de este soneto destacando los tres elementos léxico-semánticos que lo configuran: el tiempo, la naturaleza y la religión. El primero, elemento fundamental y tema principal del poema, ya que está dedicado a "un beau soir", se extiende y está presente en todas las estrofas. En el primer cuarteto encontramos los siguientes términos: "un beau soir", "un soir", "du saint jour"; en el segundo: "le soleil descend en paix du ciel" haciendo clara referencia al atardecer; en el primer terceto sólo encontramos "ce moment" que no es otro que "le soir" y por último en el segundo terceto, destacamos dos claros marcadores temporales, "toute l'année" y "à chaque heure". Por lo que respecta al segundo elemento, la Naturaleza, ésta está presente de forma muy activa en todo el poema. Sainte-Beuve personifica cada uno de los elementos naturales que menciona, realzando de este modo su poder y su fuerza. En el primer cuarteto, tal vez la estrofa más impactante de todo el soneto, la belleza de "le soir" le parece al poeta tan abrumadora que hasta la propia Naturaleza guarda silencio ("la Nature en prière se tait"), para respetar ese momento, como hizo la Virgen María cuando el arcángel San Gabriel vino a contarle la buena nueva. La comparación de un instante en el tiempo como es "un beau soir", que podría en cierto modo parecernos incluso indefinido (una tarde cualquiera), con La Anunciación, pasaje destacado del Evangelio de San Lucas, le confiere una singularidad excepcional que le hace alcanzar la categoría de sagrado. En el segundo cuarteto se mantienen las personificaciones: "la mer dort", "le soleil descend en paix du ciel" y el poeta nos explica que "ce grand silence", muestra de respeto, sólo puede ser roto por música celestial, representada por "I'hymne heureux du triple sanctuaire" ("triple sanctuarie" aludiendo al misterio central de la fe y de la vida cristiana, la Santísima Trinidad), es decir, "'hymne" que deriva del griego "himnus" cuyo significado es "yo canto"; y por un instrumento musical que, dado el carácter religioso del poema, no podría ser otro que "l'orgue immense", 
metáfora que representa claramente el cielo y cuya música viene identificada con un elemento propio de la Naturaleza, al igual que los anteriores personificado "gronde un tonnerre éternel". En estas dos estrofas es muy evidente el sentido del oído. El léxico está relacionado directamente con la antítesis silencio-ruido: "paisible, en prière, se tait, muette, écoutait, dort, en paix, silence, entend, hymne, orgue, gronde, tonnerre". En los tercetos hay casi una ausencia total de elementos naturales, únicamente rota por el complemento que cierra el soneto "un divin firmament", que más que un elemento propio de la naturaleza, nos hace pensar en la espiritualidad y la eternidad que para la religión católica representa el firmamento. El último elemento que indicábamos al principio es la religión, que confiere un cariz santo y sagrado a todo el poema. Las referencias religiosas son muy numerosas y están evidenciadas por verbos, adjetivos y nombres propios o comunes: "solennel, saint jour, en prière, Marie à genoux, Gabriel, en paix, le ciel, l'hymne, triple sanctuaire, l'orgue, éternel, tête baissée, sainte pensée, adorer, au sein d'Abraham, ton âme baignée de prière, divin firmament" ${ }^{\prime 11}$.

\section{OtROS TRADUCTORES DE WORSWORTH: ÉMILE LEGOUIS Y ANDRÉ KOSZUL}

\section{1. Émile Legouis (1861-1937)}

Nacido en Honfleur, en la región de Calvados, Émile Legouis fue profesor de literatura inglesa en La Sorbona, donde sobresalió por la sensibilidad erudita de sus comentarios y el arte de sus traducciones. En cuanto a su producción literaria hemos de destacar monografías, como Edmund Spenser (1923), y antologías entre las que sobresale William Wordsworth (1928), en la que incluyó traducciones en verso. Dirigió, además, la publicación de Contes de Canterbury en francés (Alcan, 1908) y

C'est un beau soir, un soir paisible et solennel; Era una hermosa tarde, apacible y solemne; à la fin du saint jour, la Nature en prière del santo día al final, la Natura que reza se tait, comme Marie à genoux sur la pierre, qui tremblante et muette écoutait Gabriel: como María, se calla, arrodillada en piedra, que temblorosa y muda a Gabriel escuchara:

La mer dort; le soleil descend en paix du ciel; $\quad$ el mar duerme; el sol desciende en paz del cielo; mais dans ce grand silence, au-dessus et derrière, pero en el gran silencio, por encima y detrás, on entend l'hymne heureux du triple sanctuaire, se oye el himno alegre del triple santuario, et l'orgue immense où gronde un tonnerre éternel. y el gran órgano donde gruñe un trueno eterno.

Ô blonde jeune fille, à la tête baissée,

¡Oh, jovencita rubia, de cabeza agachada qui marches près de moi, si ta sainte pensée que andas junto a mí, si tu santo pensar semble moins que la mienne adorer ce moment, menos que el mío parece adorar este instante,

C'est qu'au sein d'Abraham vivant toute l'année, en el seno de Abraham viviendo todo el año ton âme est de prière, à chaque heure, baignée; tu alma en todo momento, impregnada de rezos; c'est que ton cœur recèle un divin firmament. pues tu corazón teme un sacro firmamento. 
escribió la primera parte de Histoire de la littérature anglaise, que vería la luz en $1924^{12}$.

De su antología sobre William Wordsworth, hemos seleccionado la traducción del conocido poema "Daffodils", también conocido con el título "I wandered lonely as a cloud ", escrito por Wordsworth en 1804 y publicado en 1815 en sus Collected Poems, cuatro estrofas de seis versos cada una, en el que el poeta despliega toda la emoción, el amor y la alabanza de un campo en flor con narcisos ${ }^{13}$; el poema presenta el siguiente esquema: la rima de cada estrofa es "riche croisée" en sus primeros cuatro versos $(A B A B)$, rimando los dos últimos con rima "plate" (CC), ofreciendo los numerosos encabalgamientos un flujo continuo de expresiones sin pausa. La traducción de Legouis presenta la misma estructura: cuatro estrofas compuestas, cada una, de seis versos decasílabos, los cuatro primeros con rima "riche croisée" (ABAB), y terminando la estrofa en dos versos de rima "plate" (CC), veámoslo:

\author{
DAFFODILS \\ I wandered lonely as a cloud \\ that floats on high o'er vales and hills, \\ when all at once I saw a crowd, \\ a host, of golden daffodils; \\ beside the lake, beneath the trees, \\ fluttering and dancing in the breeze. \\ Continuous as the stars that shine \\ and twinkle on the Milky Way, \\ they stretched in never-ending line \\ along the margin of the bay: \\ ten thousand saw I at a glance, \\ tossing their heads in a sprightly dance. \\ The waves beside them danced, but they \\ out-did the sparkling waves in glee: \\ a poet could not but be gay, \\ in such a jocund company: \\ I gazed - and gazed - but little thought \\ what wealth the show to me had brought:
}

\footnotetext{
${ }^{12}$ La segunda parte es obra del autor Louis Cazamian, y ha sido considerada durante mucho tiempo obra de referencia de los anglicistas franceses,; añadamos que muchas de las obras de Émile Legouis fueron traducidas al inglés.

${ }_{13}$ Sabido es que el poeta escribió "Daffodils" en un día de tormenta en primavera, mientras caminaba junto a su hermana Dorothy cerca del Lago Ullswater, imaginando que los narcisos estaban bailando e intentando reunirse para disfrutar de la naturaleza, de las montañas y de los campos.
} 
For oft, when on my couch I lie in vacant or in pensive mood, they flash upon that inward eye which is the bliss of solitude; and then my heart with pleasure fills, and dances with the daffodils.

Traducción de Émile Legouis (1895):
LES NARCISSES
J'errais comme un nuage solitaire qui flotte au loin sur les monts et les prés, quand tout à coup je vis luire sur terre un bataillon de narcisses dorés.
Au bord d'un lac où la vague se brise ils frissonnaient et dansaient à la brise.
Comme l'œil voit se fondre dans les cieux les astres d'or, fleurs de la Voie Lactée, les fleurs du lac en ligne illimitée brillaient au bord des flots capricieux. Et je voyais, courbant leurs tiges lisses, danser au vent des milliers de narcisses.
Les flots joyeux, moins joyeux que les fleurs, les flots dansaient avec un air de fête. Je regardais: pouvais-je, moi poète, rester morose avec ses gais danseurs? Et j'emportai dans mon âme ravie, sans le savoir, un trésor pour la vie.
Triste ou sentant la tristesse venir, combien de fois, l'esprit rêveur ou sombre, j'ai vu depuis danser les fleurs sans nombre avec les yeux charmés du souvenir! Aussitôt plein de nouvelles délices mon cœur joyeux danse avec les narcisses.

El título, "Les Narcisses" es una palabra sencilla que nos recuerda la llegada de la primavera, cuando el campo está lleno de narcisos: los narcisos son flores de color amarillo, de forma sorprendente y suave fragancia; un ramo de narcisos simboliza la alegría y la felicidad de la vida, por lo tanto, el tema del poema es el conjunto de las emociones humanas inspiradas en la naturaleza que podamos haber descuidado debido a 
nuestras ajetreadas vidas. Los narcisos implican el renacimiento, un nuevo comienzo para los seres humanos, bendecido con la gracia de la naturaleza y el poeta le da la bienvenida en marzo, mes del comienzo de la primavera, y lo hace con un lenguaje descriptivo y con una redacción sencilla y melodiosa.

En el primer verso el traductor hace buen uso de la personificación y el símil. El poeta se ve a sí mismo como una nube (símil) vagando por el cielo: "J'errais comme un nuage solitaire" y el segundo y tercer versos "Qui flotte au loin sur les monts et les prés / quand tout à coup je vis luire sur terre" son la personificación de un objeto inanimado (nube) que posee la calidad de un ser humano y le permite ver los narcisos. El cuarto verso "Un bataillon de narcisses dorés" es una exageración y una hipérbole, que expresada en números en el poema de Wordswoth, "Ten thousand" describe la escena de diez mil narcisos juntos, reforzada con las imágenes de los versos 9,12 y 21 al hablar de "ligne illimitée", "des milliers de narcisses" y "les fleurs sans nombre". La aliteración del sonido /s/ y /z/ de los versos 5 y 6 nos hace llegar la música ("la brise") con la que los narcisos bailan. Queremos destacar en estas dos estrofas, en primer lugar la presencia continua de tres elementos de la naturaleza: el aire, la tierra y el agua; en segundo lugar la importancia conferida al sentido de la vista, necesario para captar toda la luz y el color desplegados en estos versos y en tercer lugar la persistente repetición del verbo "danser" en todas las estrofas del poema, para expresar el movimiento de los narcisos. Con respecto al primero, el poeta alude al aire en "flotte, brise, cieux, vent"; a la tierra en "monts, prés, terre"; y al agua en "lac, vague, lac, flots (repetido varias veces)". La luz y el color de los narcisos que alegran el campo y al poeta están presentes a través de los siguientes términos: "je vis, luire, dorés, l'œil voit, d'or, brillaient, je voyais" y de la metáfora "fleurs de la Voie Lactée" ya que nos hace pensar en los narcisos como estrellas que brillan en el cielo.

La tercera estrofa es la eclosión de la felicidad. El poeta no puede de ningún modo sentirse indiferente ante la alegría emanada por los elementos de la naturaleza, personificados en este caso, ("les flots joyeux, moins joyeux que les fleurs", "air de fête", "ses gais danseurs"), él mismo se hace esa pregunta retórica "pouvais-je, moi, poète, rester morose avec ses gais danseurs?", evidentemente no, por lo que se deja contagiar por esa alegría haciendo que su alma se sienta "ravie" y guardando en su corazón este sentimiento como si de un tesoro se tratase ("un trésor pour la vie").

Al comentar la última estrofa retomemos el primer verso del poema: "J'errais comme un nuage solitaire". El poeta nos presenta en primera persona su estado de ánimo: “j'errais", es decir, anda vagando de un lado a otro sin rumbo ni destino, triste y solitario como una nube en el cielo, alejado 
de todo y de todos. De pronto presencia un gran espectáculo: los narcisos en flor cubren montes y prados y esta visión le suscita todo tipo de agradables sentimientos, contento, júbilo, entusiasmo y bienestar. En la última estrofa, el poeta nos expresa la fuerza del recuerdo de este momento, que guardará en su corazón toda la vida, de modo que en un momento de soledad o tristeza, "triste ou sentant la tristesse venir", cuando de nuevo su alma se sienta triste y solitaria, "l'esprit rêveur ou sombre", podrá recordar y rescatar los sentimientos que experimentó ante la contemplación de un espectáculo tan maravilloso como fue ver los narcisos bailar con la brisa y brillar al sol. De este modo, al recuperar estas sensaciones el poeta participará de la alegría de los narcisos, pues, como nos explica en el último verso del poema, su corazón bailará con ellos ${ }^{14}$.

\section{2. André Koszul (1878-1956)}

Nacido en Roubaix, Koszul fue catedrático de universidad y doctor en letras por la universidad de Strasbourg, en la que enseñaría durante más de

\footnotetext{
${ }^{14}$ En nuestra traducción al español de la traducción francesa de Legouis, hemos utilizado el endecasílabo, más frecuente en español, tratando de respetar la rima asonante: LOS NARCISOS

Vagaba como una nube errante que flota sobre los montes y prados, y vi brillar en tierra de repente un batallón de narcisos dorados. Junto al lago donde la ola se riza temblaban y bailaban con la brisa.

Igual que iris ve fundirse en el cielo Astros de oro, flores de la Vía Láctea, flores del lago en infinita recta brillaban en las olas con anhelo. Veía, al curvarse sus tallos lisos, bailar al viento miles de narcisos.

Las olas felices, aún más las flores, las olas bailaban como una opereta, yo miraba: ¿podía acaso un poeta, estar triste con tales bailarines? y guardé , pues, en mi alma emotiva, sin saberlo, un tesoro de por vida

Triste o sintiendo la tristeza llegar, ¡cuántas veces, perdido y pesimista volví a ver bailar flores infinitas con el recuerdo cautivado del mirar! Pronto, lleno de nuevos regocijos baila mi alma feliz con los narcisos.
} 
treinta años. En 1910 llevó a cabo una "Thèse d'État" con el título de La Jeunesse de Shelley, autor del que posteriormente (1929) publicaría en francés una selección de poemas. Koszul dirigió varias colecciones: "Shakespeare", editada en un primer momento por la firma inglesa J. M. Dent ${ }^{15}$ y más tarde por la francesa Les Belles Lettres, en las que él mismo propuso traducciones al francés de las comedias de Shakespeare; asimismo, "Théâtre anglais de la Renaissance". Koszul destacó sobre todo por su magnífico talento como traductor, y, como tal, en sus traducciones procuraba mantener una fidelidad de impresión más que una equivalencia literal, tratando de conservar el ritmo del original; era corriente oírle decir que la traducción era para él "la dixième Muse". Por último, su Anthologie de la littérature anglaise (2 volúmenes) fue publicada en 1912 y 1916 respectivamente. De este autor hemos seleccionado la traducción del poema de Wordsworth "A Few Miles Above Tintern Abbey" (1798):

FIVE years have past; five summers, with the length of five long winters! and again I hear these waters, rolling from their mountain-springs with a soft inland murmur. -- Once again do I behold these steep and lofty cliffs, that on a wild secluded scene impress thoughts of more deep seclusion; and connect the landscape with the quiet of the sky. [...] These beauteous forms, through a long absence, have not been to me as is a landscape to a blind man's eye: but oft, in lonely rooms, and 'mid the din of towns and cities, I have owed to them in hours of weariness, sensations sweet, felt in the blood, and felt along the heart; and passing even into my purer mind, with tranquil restoration: -- feelings too of unremembered pleasure: such, perhaps, as have no slight or trivial influence on that best portion of a good man's life, his little, nameless, unremembered, acts of kindness and of love. Nor less, I trust, to them I may have owed another gift, of aspect more sublime; that blessed mood, in which the burthen of the mystery, in which the heavy and the weary weight of all this unintelligible world,

${ }^{15} \mathrm{~J}$. M. Dent and Company fue fundada en 1888 por Joseph Malaby Dent y pasó a denominarse J.M. Dent \& Sons en 1909. 
is lightened: -- that serene and blessed mood, in which the affections gently lead us on, -until, the breath of this corporeal frame and even the motion of our human blood almost suspended, we are laid asleep in body, and become a living soul: while with an eye made quiet by the power of harmony, and the deep power of joy, we see into the life of things.

If this be but a vain belief, yet, oh! how oft -in darkness and amid the many shapes of joyless daylight; when the fretful stir unprofitable, and the fever of the world, have hung upon the beatings of my heart -how oft, in spirit, have I turned to thee, o sylvan Wye! thou wanderer thro' the woods, how often has my spirit turned to thee! [....] To me was all in all. -- I cannot paint what then I was. The sounding cataract haunted me like a passion: the tall rock, the mountain, and the deep and gloomy wood, their colours and their forms, were then to me an appetite; a feeling and a love,

that had no need of a remoter charm, by thought supplied, nor any interest unborrowed from the eye. -- That time is past, and all its aching joys are now no more, and all its dizzy raptures. Not for this faint I, nor mourn nor murmur, other gifts have followed; for such loss, I would believe, abundant recompense. For I have learned to look on nature, not as in the hour of thoughtless youth; but hearing oftentimes the still, sad music of humanity, nor harsh nor grating, though of ample power to chasten and subdue. And I have felt a presence that disturbs me with the joy of elevated thoughts; a sense sublime of something far more deeply interfused, whose dwelling is the light of setting suns, and the round ocean and the living air, and the blue sky, and in the mind of man; a motion and a spirit, that impels all thinking things, all objects of all thought, and rolls through all things. Therefore am I still a lover of the meadows and the woods, and mountains; and of all that we behold 
from this green earth; of all the mighty world of eye, and ear, -- both what they half create, and what perceive; well pleased to recognise in nature and the language of the sense, the anchor of my purest thoughts, the nurse, the guide, the guardian of my heart, and soul Of all my moral being.

Nor perchance, $[\ldots]$

\section{Traducción de André Koszul (1919):}

VERS ECRITS A QUELQUES MILLES AU-DESSUS DE TINTERN ABBEY Cinq années sont passées - cinq étés et cinq longs, longs hivers! - Et voici que j'entends à nouveau ces eaux qui, descendues des monts, gardent encore cette intime douceur des voix du haut pays. Je contemple à nouveau ces fiers rochers abrupts qui jettent sur ces lieux agrestes et secrets, un air de solitude plus profonde, et semblent joindre à la paix des champs la grande paix des cieux. [...] Ces formes de beauté n'ont pas été pour moi, pendant ma longue absence, ce qu'est un paysage à l'œil qui ne voit plus : mais souvent dans la chambre vide, au cœur des bruits incessants de la ville, elles m'auront valu, aux heures de langueur, ces douces impressions qu'on sent courir, avec le sang, jusqu'au cœur même, et pénétrer enfin le plus pur de notre âme, pour la régénérer paisiblement; bien plus, je leur ai dû ce sens de bonheurs oubliés, ce sens qui peut laisser sa trace forte et sûre dans ce qu'a de meilleur la vie d'un honnête homme, sur ces mille petits actes immémorés de tendresse et d'amour. Et je veux croire aussi qu'elles m'auront valu cet autre grand bienfait, le sublime bienfait de ces instants bénis où l'on sent tout à coup le grand poids du mystère, le fardeau écrasant, le fardeau épuisant de l'inintelligible énigme de ce monde, devenir plus léger... L'humeur sainte et sereine où, doucement conduit par l'instinct de l'amour, le souffle de ce corps grossier suspendu presque, le mouvement du sang dans ces veines mortelles presque arrêté, on se trouve comme endormi dans la chair, et vivant la seule vie de l'âme cependant que d'un œil calmé par le pouvoir des harmonies du monde, et le plus grand pouvoir 
de la joie, on pénètre au cœur même des choses! Peut-être n'est-ce là qu'un mirage, mais quoi? Je sais que dans la nuit, ou parmi les fantômes qui passent dans ce morne jour, lorsque l'émoi, l'agitation vaine, et la fièvre du monde, ralentissaient déjà mes battements de cœur, maintes fois, en esprit, je me tournai vers toi. Ô sylvaine rivière, errante sous les arbres, maintes fois mon esprit s'est retourné vers toi. [...] La nature alors m'était tout - je ne puis dire ce que j'étais alors. La cascade qui gronde me hantait comme une passion; le haut rocher, la montagne, et le bois profond et ténébreux, leurs formes, leurs couleurs, oui, alors, j'avais soif de tout cela ; je les sentais, je les aimais; point ne m'était besoin du charme plus lointain que fournit la pensée, ou d'un plaisir quelconque qui n'aurait point passé par les yeux. II n'est plus, l'heureux temps dont les joies âpres faisaient souffrir, dont les ravissements donnaient presque un vertige! Mais je ne me plains pas, je ne murmure pas; d'autres dons ont suivi, qui, je voudrais le croire compensent amplement ma perte. Oui, j'ai appris à voir notre univers, non dans l'insouciance de la jeunesse, mais l'oreille aux écoutes de ce chant triste et doux qu'y fait l'humanité musique très discrète, et pourtant toute empreinte d'un charme purificateur. Ainsi j'y trouve une présence auguste, et de hautes pensées m'émeuvent de leur joie - un sublime soupçon qu'il est, intimement mêlé au monde, un Être, hôte mystérieux de l'occident en feu, de l'ample sein des mers, de l'air qui vivifie, hôte du ciel d'azur, hôte de l'âme humaine, un mouvement et un esprit, qui régit tout, l'homme pensant, comme l'objet de sa pensée, un Être épars dans tous les êtres. C'est ainsi que, toujours amoureux des prairies et des bois, et des monts, et de tout ce que nous contemplons sur cette verte terre, et de l'immense empire de l'oreille et de l'œil - par eux demi perçu, demi créé par eux - j'aime aussi reconnaître, dans la nature et dans le message des sens, le fondement de mes plus purs pensers, le maître le guide, le gardien de mon cœur, l'âme même 
de tout ce que je crois et de tout ce que j'aime. $[. . .]^{16}$

\footnotetext{
${ }^{16}$ Veamos nuestra traducción española: VERSOS ESCRITOS A UNAS MILLAS MÁS ARRIBA DE LA ABADÍA DE TINTERN ¡Han pasado cinco años - cinco largos veranos y sus largos inviernos! - De nuevo vuelvo a oír las aguas que, al bajar del monte, aún guardan la íntima dulzura de una voz en la cumbre. De nuevo veo estas rocas altivas y abruptas que dan a estos parajes, agrestes y secretos, un aire solitario más profundo, y parecen unir la paz del cielo a la quietud del campo. [...] Estas hermosas formas durante mi amplia ausencia, no han sido para mí nada más que un paisaje que no se vuelve a ver: mas, en mi cuarto a veces, vacío pero en el centro de los ruidos del pueblo, han sido para mí, en los momentos tristes, las dulces sensaciones que fluyen por la sangre llegando al corazón, y alcanzando hasta lo último, más puro de nuestra alma, para regenerarla dulcemente; y aún más, les debo ese sentido de gozos olvidados, sentido que deja su huella fuerte y firme en lo que es más preciado en la vida de un hombre, en esos miles de actos, pequeños y olvidados de ternura y de amor. También quiero creer que ellas me han regalado este otro gran placer, el sublime placer de momentos benditos en que al pronto sentimos el peso del misterio, la carga aplastante, la carga agotadora del ininteligible enigma de este mundo, hacerse más ligero... ¡Humor santo y sereno en el que, conducido por la intuición de amor, de este cuerpo basto casi colgado, el hálito el flujo de la sangre por mis venas mortales está casi parado, como adormecido en la carne, y viviendo del alma la vida única mientras que con mirada por el poder calmada de los ritmos del mundo, y el poder aún más grande de la alegría, se entra en el alma de todo! Quizá sólo espejismo, pero, ¿qué más me da? Yo sé que por la noche, o hasta entre los fantasmas que hay en este día lúgubre, cuando el desasosiego, la vana agitación, y la fiebre del mundo conseguían reducir mis rápidos latidos, muchas veces, en ánimo, me volví hacia ti. ¡Oh selvático río, que yerra bajo los árboles, muchas veces mi espíritu se volvió hacia ti! [...] La Natura entonces para mí lo era todo no sé que era yo entonces. La cascada que gruñe cual pasión me asediaba; el gran acantilado la montaña, el bosque profundo y tenebroso,
} 
Comentario del poema: el título hace referencia a un lugar real, Tintern Abbey, una abadía en ruinas abandonada en 1536 y situada al lado del río Wye al oeste de Inglaterra. Se trata de una composición de ciento sesenta versos, escrita en primera persona y en la que el poeta nos muestra su propia personalidad. El poema de Wordsworth está compuesto en pentámetros yámbicos ${ }^{17}$, por lo tanto sin rima. Su estilo es, pues, muy fluido y natural, hecho que facilita la lectura, haciéndola más ágil, como si se tratase de una obra en prosa, aunque la estructura poética está perfectamente construida. En la traducción al francés André Koszul elije

sus formas, sus colores: entonces tenía sed

de todas esas cosas, sintiéndolas, amándolas nada necesitaba de un encanto lejano

que a la mente nutriese, ni cualquier placer otro

que se hubiese expuesto a mis ojos.jPasó

el feliz tiempo en el que las agrias alegrías

dolían y sus encantos casi vértigo daban!

Pero no me lamento, ni tampoco murmuro;

otros dones llegaron, que, quisiera creerlo

compensan ampliamente mi pérdida. Aprendí

sí, a ver nuestro mundo no desde la inconsciencia

de la juventud, sino oyendo el canto suave

y triste que entona la humanidad entera -

música muy discreta, y sin embargo huella

de un encanto puro. Así, encuentro en él

la augusta presencia, y altos pensamientos

de alegría me conmueven - una magna sospecha

que está íntimamente unida al mundo, un Ser,

un huésped misterioso del ardiente occidente,

del gran seno del mar, del aire que da vida,

huésped del azul cielo, huésped del alma humana,

movimiento y ánima que lo dirige todo,

el pensante hombre, como de su pensar objeto,

un Ser disperso en todos los seres. Y, por ello,

de praderas y bosques siempre está enamorado,

de montes y de todo lo que admirar podemos

sobre esta verde tierra, y del inmenso imperio

del oído y de la vista - por ellos percibido,

medio creado por ellos - reconocer me gusta,

en la naturaleza y en todos los sentidos

la base de mis más puras ideas, el maestro,

guía y guardián de mi pecho, hasta de mi propia alma

de todo lo que creo, de todo lo que amo.

${ }^{17}$ Digamos, a modo de recordatorio, que el pentámetro yámbico es un tipo de verso de cinco pies, cada uno de los cuales suele estar compuesto de dos sílabas, no acentuada y acentuada, con una sílaba opcional no acentuada al final.; el poema no tiene rima.

Hikma 8 (2009), 35-62 
"des alexandrins à rime libre", es decir versos de doce sílabas sin rima, separados por la cesura en dos hemistiquios de seis sílabas.

Tintern Abbey es una declaración del joven Wordsworth en la que nos introduce el siguiente tema: el recuerdo de la comunicación pura con la naturaleza durante su juventud y el regocijo ya en la edad adulta, cuando dicha comunicación se ha perdido, de saberse poseedor de la capacidad de "mirar a la naturaleza" y escuchar "la humanidad", es decir, recrear su mirada en la naturaleza y sentirla unida a la vida del hombre. El poema comienza con la declaración del poeta de que cinco años han pasado desde la última vez que visitó aquel lugar, que para el poeta representan casi una eternidad (cinq années..., - cinq étés, cinq longs, / longs hivers) y nos dibuja una escena tranquila y rústica en la que nos describe todo lo que ve y su efecto sobre él mismo: el poeta relaciona el pasado con el presente ("à nouveau") al describir todos esos elementos naturales que le hacen revivir aquellos sentimientos de calma, paz, dulzura y soledad absolutas. (En la traducción al francés estos sentimientos están claramente reforzados por la aliteración de sonidos sibilantes). Se deja caer sobre un árbol y observa los árboles frutales con los frutos aún sin madurar, ve el humo de las chimeneas elevarse entre los árboles y dirigirse hacia lo profundo del bosque. Esta visión le hace pensar en el tiempo en que estuvo alejado de aquel paisaje y en los recuerdos que lo acompañaron durante esta "larga" ausencia: cuando el poeta se encontraba solo en la gran ciudad, aquellos recuerdos le proporcionaron agradables sensaciones "douces impressions / qu'on sent ocurrir, avec le sang, jusqu'au cœur même, / et pénétrer en fin le plus pur de notre âme, / pour la régénérer paisiblement...", clara evocación de un éxtasis discreto manifestado con precisión pero en su justa medida. Dichas sensaciones enriquecían su mente con calma renovadora y aportaban, de manera inconsciente, amabilidad y amor a su forma de actuar. Ahora al contemplar aquella escena, los recuerdos pasados le visitan de nuevo, haciéndole sentir una amarga alegría al revivirlos y una inaudita felicidad al saber que aquella experiencia presente le proporcionará infinitos recuerdos para los años venideros. El autor se reconoce diferente a aquel joven que vivía rodeado de montañas y riachuelos. En aquellos días, la naturaleza constituía su único mundo: las cascadas, las montañas y los bosques conformaban sus pasiones y sus deseos. Ahora, esos días han pasado, aunque no lo lamenta, pues a pesar de no poder recuperar su pasada relación con la naturaleza, esta pérdida ha sido compensada con otros dones maduros, pues dicha madurez le permite "oír la música de la humanidad": "D'autres dons ont suivi, qui, je voudrais le croire / compensent amplement ma perte. Oui, j'ai appris / à voir notre univers, non dans l'insouciance / de la jeunesse, mais l'oreille aux écoutes / de ce chant triste et doux qu'y fait l'humanité", y sentir la presencia de algo más sutil, 
poderoso y fundamental en la luz del sol, en el océano, en el mismo aire, incluso en la mente del hombre. El poeta concibe esta energía como un movimiento y un espíritu que impulsa todos sus pensamientos "un mouvement et un esprit, qui régit tout, / l'homme pensant, comme l'objet de sa pensée, / un Être épars dans tous les êtres", por ello, sigue enamorado de la naturaleza, de las montañas, de los bosques, de los pastos y permanecen en el pensamiento, en el corazón y en el alma de ese "moral being" (ser moral), que no es otro que él mismo. Además añade que aunque no lo sintiera de este modo o no comprendiese lo que está sintiendo, él se sentiría bien pues está en compañía de su hermana "dear, dear Sister", que también es su "dear, dear, Friend" y en cuya voz y actitud rememora su infancia. Este hecho le hace elevar una oración a la naturaleza, pues tiene la certeza de que ella nunca traicionará al corazón que tanto la amó "Nature never did betray / The heart that loved her". El poeta continúa incitando a los elementos de la naturaleza, a la luna, al viento a que se proyecten sobre la imagen de su hermana, de este modo, en años posteriores, cuando ella esté triste 0 aterrada, el recuerdo de esta experiencia le ayudará a olvidar estos sentimientos, e incluso cuando él muera, ella podrá recordar el amor con el que él adoró a la naturaleza: lo que los bosques significaron para él, no sólo por sí mismos sino por el hecho de que su hermana también estaba a su lado.

Como acabamos de ver, estamos ante un poema que no es más que un monólogo dirigido hacia el mismo poeta y excepcionalmente a su hermana, a la luna o al espíritu de la naturaleza. El lenguaje destaca por su simplicidad y sinceridad y huye de la ostentación, pues la única preocupación el poeta es hablar, con franqueza, desde el corazón. Las imágenes del poema se limitan al mundo natural en el que el poeta se mueve, y las metáforas se enmarcan en tres campos claros: el propio yo del poeta, el tema náutico (el recuerdo es "the anchor" del pensamiento del poeta) y la arquitectura (la mente es la "mansion" del recuerdo). De igual modo encontramos referencias religiosas: "instants bénis", "mystère", "humeur sainte et sereine"... Si bien la actual forma de la abadía no está presente en el poema, el poeta sí nos trasmite la idea de "abadía" como lugar sagrado y consagrado al espíritu. Dicha idea se ve reforzada por la descripción de la fuerza del sol, pues en su mente se produce una inexorable unión de conceptos: la idea de Dios, la de la naturaleza y la de la mente humana. Conceptos insistentes y destacados en toda la poesía de Wordsworth.

\section{CONCLUSIONES}

Es indiscutible que merced al especial interés que los poetas "lakistas", y en concreto William Wordsworth, despiertan en Sainte-Beuve 
esta poesía íntima y familiar se vaya haciendo hueco poco a poco en la literatura francesa hasta adquirir un auge singular y la aceptación de la crítica del país galo. Por ello es necesario reconocer a Sainte-Beuve su magnífica labor como introductor en Francia de las nuevas tendencias románticas y de los nuevos aires renovadores que ya se vislumbraban en la poesía de estos autores "lakistas", todo ello debido a las traducciones que realizará de Coleridge y de Wordsworth, del mismo modo que a los poemas de creación propia donde ya se vislumbra el gusto por la sencillez, la cotidianeidad y la intimidad, características que definieron la obra de los poetas del "The Lake District". Sainte-Beuve no posee las dotes ni la técnica de Victor Hugo, su inspiración no tiene ni la magnitud ni el esplendor del gran lirismo romántico, él mismo reconocerá sentirse inferior a sus maestros y a sus rivales, de ahí, que consciente de sus limitaciones, adopte un género a su medida y ofrezca el modelo de una poesía familiar, a menudo prosaica, siguiendo los pasos del estilo aparentemente "artless" propio de Wordsworth, pero rica en anotaciones íntimas y en confidencias veladas, pues el mensaje poético está más en el contenido que en la forma. A través de su poesía, Sainte-Beuve nos desvela a un analista del alma humana y a un humanista (recuerdos de la Antigüedad, de la Edad Media, de La Pléiade). Su poesía destaca por un lirismo confidencial, ya que desvela un alma sutil, sensible, replegada sobre sí misma. Con Sainte-Beuve, el "mal du siècle" pierde su grandeza, pero se hace más íntimo ya que consigue matizarlo con la incorporación de detalles de la vida cotidiana y de un decorado familiar. Por otra parte, Sainte-Beuve practicará, en pleno romanticismo, el género íntimo (al que más tarde llamarán "intimismo") y del que se convertirá en precursor: su tristeza obsesiva, orienta la melancolía romántica hacia el "spleen" de Baudelaire (sus escenas de la calle son como un primer esbozo de los Tableaux parisiens en Les Fleurs du Mal). De igual modo, su gusto por el humilde detalle prosaico anuncia la poesía familiar de François Coppée. Incluso Verlaine y los simbolistas apreciarán sus "paysages d'âme", su sensibilidad secreta y melódica. A pesar de ello, Sainte-Beuve, tras su "crise intérieure" de los años 1834-35, renuncia a su faceta como poeta para dedicarse a la crítica. Con el tiempo encontrará un lugar, aunque no la gloria, entre los grandes creadores de su generación gracias al prestigio de su arte y a la importante repercusión que sus traducciones de los autores 'lakistas' y sus poesías simples y sentidas tuvieron en la literatura francesa del siglo XIX y como hemos podido comprobar, también en grandes traductores del siglo $\mathrm{XX}$.

REFERENCIAS BIBLIOGRAFICAS

Biedermann, Alfred: Le romantisme européen, Tome I et Tome II. Paris: Classiques Larousse, 1972. 
DANBY, John F.: The Simple Wordsworth: Studies in the Poems 1797-1807, London: Routledge and Kegan Paul, 1968.

DE Mirecourt, Eugène: Sainte-Beuve, cinquième édition, éd. Gustave Havard, Paris, 1856.

DE QUINCEY, Thomas: «Essays on Wordsworth», David Nichol Smith. (ed.). Wordsworth. Poetry and Prose. With Essays by Coleridge, Hazlitt, de Quincey. Oxford: Clarendon Press, 1960.

DURANT, Geoffrey: Wordsworth and the Great System: A Study of Wordsworth's Poetic Universe. Cambridge University Press, 1970.

Groom, Bernard: The Unity of Wordsworth's Poetry. New York: St. Martin's Press, 1966.

JUNG, Sandro: "Wordsworth's "Tintern Abbey" and the tradition of the "hymnal" ode". Miscelánea: a journal of English and American studies 34 (2006), pp. 63-74.

LEGOUIS, Émile: Wordsworth, William, 1770-1850; Vallon, Annette; Vallon family, Publisher [Hamden, Conn.] Archon Books, Book from the collections of University of California, 1922.

- La jeunesse de William Wordsworth 1770-1798; étude sur le "Prélude », Paris: G. Masson, 1896. et CAZAMIAN, L., Histoire de la littérature anglaise, Paris: Hachette, 1924.

MARVELL, Andrew: The critical heritage, London: Publisher Routledge, 1995.

MASON, Michael: Lyrical Ballads, London and New York: Longman, 1994.

MOORMANN, Mary: William Wordsworth: The Early Years, Oxford: Clarendon Press, 1957.

POLET, Jean-Claude: Patrimoine littéraire européen : anthologie en langue française, editorial De Boeck \& Larcier, Département De Boeck Université, Paris-Bruxelles, 1996.

SAINTE-BeuVE, Charles-Augustin: Poésies complètes de Sainte-Beuve, Paris: Charpentier Libraire-Éditeur, 1845. Vie, poésies et pensées de Joseph Delorme, Paris: Michel Lévy Frères, Libraires-Éditeurs, 1863. , Mis venenos, traducción de Fátima Sainz y Maryse Privat, Colección / Clásica Artemisa-Ediciones, 2007.

WORDSWORTH, William: Lyrical Ballads, 1800, 1802: Edición Bilingüe, Madrid: Ediciones Hiperion, S.L. 1999. 